\title{
Caracterización e identificación de la geomorfología (ambientes y unidades geomorfológicas) en la plancha 121 - Cerrito, Colombia, aplicado a movimientos en masa, escala 1:100.000
}

\author{
Oscar Enrique Forero-Ospino ${ }^{1 *}$, William Fernando Duarte-Delgado ${ }^{2}$
}

\begin{abstract}
DOI: http://dx.doi.org/10.18273/revbol.v41n2-2019002 @c) (1)
\end{abstract}
Forma de citar: Forero-Ospino, O.E., y Duarte-Delgado, W.F. (2019). Caracterización e identificación de la geomorfología (ambientes y unidades geomorfológicas) en la plancha 121 - Cerrito, Colombia, aplicado a movimientos en masa, escala 1:100.000. Boletín de Geología, 41(2), 35-44. DOI: 10.18273/revbol.v41n2-2019002

Material suplementario electrónico: La versión en línea de este artículo contiene MATERIAL SUPLEMENTARIO

\section{RESUMEN}

Esta investigación centra su trabajo en la identificación de unidades geomorfológicas dentro del área de la plancha 121 Cerrito, que corresponde al municipio de Cerrito en el departamento de Santander, Colombia, tomando como criterio la evolución geológica. De esta misma manera, se analizan los movimientos en masa (MM) que muestran la geomorfología activa (morfodinámica) en cuestión de procesos activos como erosión pluvial, socavación y reptación, entre otros. Estos procesos afectan litologías específicas por sus propiedades físicas y químicas junto con la generación de suelos produciendo características favorables a la acumulación o a altos contenidos de agua en su estructura (suelos arcillosos). La consecuencia en épocas de precipitaciones constantes y grandes volúmenes de líquido es la generación de movimientos tales como flujos y deslizamientos. La generación de mapas de MM puede ser de gran utilidad para estudios de zonificación de amenaza y susceptibilidad por remoción de movimientos en masa, teniendo en cuenta los ambientes geomorfológicos y su distribución en toda el área de estudio.

Palabras clave: Geomorfología; Deslizamientos; Evolución geomorfológica; Andes colombianos.

\section{Identification and characterization of the geomorphology (geomorphological environments and units) of the Sheet 121 - Cerrito, Colombia, applied to landmass movements, scale 1:100.000}

\begin{abstract}
This research focuses on the identification of geomorphological units based on geological evolution in the Sheet 121 - Cerrito, Santander, Colombia, using the geological evolution as criterion. In addition analysis of landmass movements is included showing active geomorphology (morphodinamics) such as pluvial erosion, hydrodynamic scour, and creep, among others. These processes affect rocks depending on their physical and chemical properties with the generation of soils producing favorable characteristics for accumulation or high content of water in their structure (clay soils). As consequence of high rainfalls and large volumes of fluids are produced movements such as flows and slides. The development of maps showing landslides is useful to mark off hazard and susceptibility zones by landmass movements considering its geomorphological features and its distribution in the area of study.
\end{abstract}

Keywords: Geomorphology; Landslides; Geomorphologic evolution; Colombian Andes.

\footnotetext{
${ }^{1}$ Departamento de Ciencias Básicas, Universidad Santo Tomás, Bucaramanga, Colombia. $\left({ }^{*}\right)$ oscar.forero@ustabuca.edu.co

${ }^{2}$ Facultad de Ingeniería Civil, Universidad Santo Tomás, Villavicencio, Colombia. williamduarte@usantotomas.edu.co
} 


\section{INTRODUCCIÓN}

A través del tiempo en términos geológicos, el relieve terrestre ha ido cambiando y evolucionando en función de los factores naturales que lo afectan, lo que más ha contribuido con dichos cambios es la intervención humana (factor antrópico). Factores naturales tales como la lluvia, la actividad tectónica y la erosión, entre otros, sumados al factor antropogénico que incluye la deforestación, la minería, el sobrepastoreo y otros usos inadecuados del suelo, han contribuido a modificar el paisaje del territorio colombiano, lo que conlleva al desarrollo de zonas de inestabilidad que dan paso a los Movimientos en Masa (MM), en diferentes formas y magnitudes, considerados como amenazas, ya que han producido pérdidas económicas y de vidas humanas en gran magnitud. Por esta razón es de crucial importancia generar mapas de unidades geomorfológicas aplicadas a MM para identificar las zonas con mayores problemáticas $\mathrm{y}$, de esta manera, empezar una evaluación de amenaza en estudios más específicos.

Por otro lado, la geomorfología como rama de la Geología que analiza las diversas formas que se encuentran sobre la superficie terrestre. Incluye la interpretación morfogenética, que busca conocer el origen de los elementos geológicos y su morfología describiendo aspectos topográficos y geométricos de las diferentes zonas de su entorno geológico. Carvajal et al. (2003a), clasifican los procesos naturales como procesos morfodinámicos externos (exógenos) e internos (endógenos), los cuales integran un conjunto de sistemas dinámicos que cambian la estructura de los paisajes naturales. El nivel de intensidad con el cual los agentes encargados de ejecutar estos procesos actúan sobre la corteza, da forma a los diversos elementos geológicos sobre la superficie terrestre y conforman un ambiente geológico específico. Dentro de los aspectos que estudia la geomorfología, se encuentran: la morfogénesis (unidades geomorfológicas), la morfodinámica (movimientos en masa) y la morfometría (análisis cuantitativo de la forma). Para el presente estudio se consideraron los primeros dos elementos de estudio de la geomorfología y el tercero se tendrá en cuenta cuando se elaboren los respectivos mapas de geomorfología y bloque diagrama.

Dentro del convenio entre la Escuela de Geología de la Facultad de Ingenierías Físico-Químicas de la Universidad Industrial de Santander y el Servicio Geológico Colombiano, denotado como convenio especial de Cooperación número 009 de 2013, se realizó la Zonificación de Amenaza Relativa por Movimientos en Masa a escala 1:100.000, para el bloque 5 compuesto por las planchas geológicas 109 - Rionegro, 110 Pamplona, 111 - Toledo, 119 - Barrancabermeja, 121 - Cerrito, 133 - Puerto Berrio, 134 - Puerto Parra, 136 - Málaga, 137 - Cocuy y 153 - Chita. Como base fundamental para la realización del estudio, se utiliza la Propuesta Metodológica Sistemática para la generación de mapas geomorfológicos analíticos aplicados a la Zonificación de Amenaza del año 2012, como antecedente del documento mencionado, generados después de la problemática en el municipio de Gramalote, departamento de Norte de Santander durante el periodo del 2010 - 2011 que, por causa del fenómeno de la niña, se deslizó el $90 \%$ de la cabecera municipal. La Escuela de Geología de la UIS realizó una selección de un equipo de estudiantes de últimos semestres, en modalidad de investigación para el desarrollo de los proyectos de tesis.

El área objeto de análisis se encuentra ajustada por la plancha 121 - Cerrito que, según el Instituto Geográfico Agustín Codazzi (IGAC) con Datum Magna Sirgas Central con límites verticales de $\mathrm{N}=1240000-$ 1280000; y con límites horizontales de $\mathrm{E}=1120000$ 1165000 , localizada entre el sureste del departamento de Santander y el sur del departamento de Norte de Santander, abarcando los municipios de Santa Bárbara, Guaca, Baraya, San Andrés, Silos, Cerrito, Concepción, Chitagá (Presidente), Piedecuesta, Cepitá y Molagavita. El área total de la cobertura de la plancha es de $1.800 \mathrm{~km}^{2}$ (ver FIGURA 1). Se identifica un límite inferior de 600 $\mathrm{msnm}$ en las regiones perimetrales del río Chicamocha al suroccidente del sector de interés y un límite superior de 4.200 msnm en los límites con Norte de Santander en la franja central del área de estudio.

Para la plancha 121 - Cerrito se ha evaluado la lluvia como detonante de MM, utilizando los mapas de zonificación climática del Atlas Climatológico de Colombia (IDEAM, 2014), como son precipitación Media Anual y Temperatura Media Anual, mostrando un desarrollo con cierta normalidad sobre un registro calendario de un año, en donde los periodos de verano o baja precipitación se producen en los meses de Diciembre, Enero y Febrero con un salto hacia los meses de Junio, Julio y Agosto; los periodos de invierno o alta precipitación se registran para la zona en los meses de Marzo, Abril y Mayo con un salto hacia los meses de Septiembre, Octubre y Noviembre. En general la plancha 121 - Cerrito registra precipitaciones anuales entre los $1.000-1.500 \mathrm{~mm} / \mathrm{año}$ en climas que van desde templados a fríos y subclimas que parten desde los muy húmedos a pluviales con temperaturas que 
oscilan entre $\operatorname{los} 15^{\circ} \mathrm{C}$ en las zonas de páramos (límites superiores entre los departamentos de Santander y Norte de Santander) hasta $\operatorname{los} 32^{\circ} \mathrm{C}$ en las zonas bajas correspondientes al margen Occidental cerca al cauce del río Chicamocha. En el sector de la plancha 121 - Cerrito se puede observar una humedad del aire relativa media anual del 75 al $80 \%$ mostrando un alto desarrollo de nubosidad y lluvias con respecto al aire saturado; junto a la velocidad media anual del viento con valores promedio de $3 \mathrm{~m} / \mathrm{s}$ de magnitud que determina la fuerza del vector, con una pequeña inclinación del vector desde el NW hacia el SE.

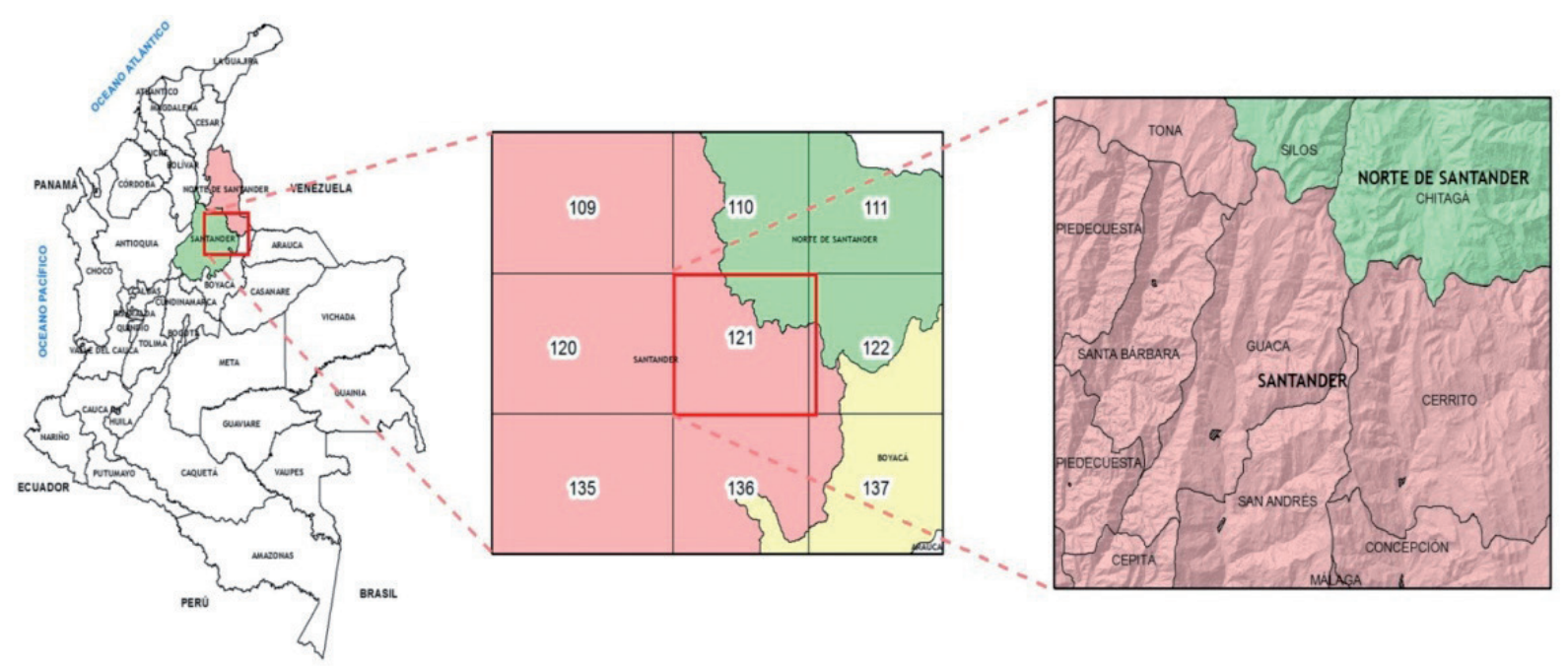

FIGURA 1. Ubicación del área del estudio con la respectiva división departamental de la plancha 121 - Cerrito.

\section{METODOLOGÍA}

Para este proyecto se toma como referencia el documento Propuesta Metodológica Sistemática para la Generación de Mapas Geomorfológicos Analíticos Aplicados a la Zonificación de Amenaza, Escala 1:100.000, elaborado por el Servicio Geológico Colombiano, en el cual se identificaron variables principales como la jerarquización geomorfológica y los ambientes morfogenéticos, entre otros.

A partir de información secundaria recolectada, este documento describe de manera general las características de la región que abarca la plancha en cuanto al contexto de los ambientes geomorfológicos, aspectos geológicos como la estratigrafía y su marco tectónico. Es importante conocer la jerarquización geomorfológica para definir, por medio de nuestra escala de trabajo, las condiciones que consigan caracterizar de manera detallada las expresiones morfogenéticas y el alcance de estas de acuerdo con el método de Verstappen y Van Zuidam (1991), que fue adoptado por Carvajal $(2002,2012)$ y Carvajal et al. (2003a, 2003b). De igual manera, se describe la estratigrafía de la plancha 121 - Cerrito para poder diferenciar algunos rasgos moldeados por el tipo de roca que conforma las unidades geomorfológicas y los efectos ante su composición mineralógica en relación con el análisis de la evolución geomorfológica.

Una variable de alta importancia es la morfodinámica, ya que ésta es la responsable de la modificación progresiva que se genera en las unidades geomorfológicas antecesoras, como, por ejemplo, en el caso de una zona de remoción de masa, la zona modificada: aquélla en donde se presentó la remoción, y la no afectada por esta: la unidad geomorfológica antecesora. Para ello, a la hora de catalogar e inventariar los movimientos de remoción en masa, se toma como referencia la metodología de Cruden y Varnes (1996), que fue adoptada por Proyecto Multinacional Andino (PMA, 2007), y que estuvo enfocada esencialmente en el tipo de movimiento y del material que lo compone. Ahora bien, debe tenerse en cuenta que la mayoría de los movimientos en masa dentro de la región que abarca la plancha 121- Cerrito pueden tener variaciones en la actualidad debido al tiempo de generación relacionado con el de la visita o inventario. 


\section{RESULTADOS}

Principales ambientes geomorfológicos en el área

El área de estudio posee características geomorfológicas bien definidas en cuatro ambientes geomorfológicos específicos (ver TABLA 1). Dentro de ellos encontramos el ambiente morfoestructural correspondiente a las geoformas generadas por la dinámica interna de la tierra o procesos endógenos, es decir, especialmente las asociadas con los plegamientos $\mathrm{y}$ el fracturamiento de las rocas. Otro ambiente importante es el denudacional, que está definido por la actividad de los procesos exógenos de la meteorización, por los procesos erosivos hídricos y por los fenómenos de transporte o de remoción en masa actuantes sobre geoformas preexistentes (SGC, 2012). El ambiente fluvial corresponde a las geoformas generadas por los procesos relacionados con la actividad de fluvial (SGC, 2012). En el ambiente glaciar se identificaron unidades geomorfológicas originadas de procesos de erosión y sedimentación producida en las partes altas de las cadenas montañosas o en zonas periglaciares, las cuales generaron grandes cantidades de sedimentos que fueron acumulados en las laderas adyacentes a esas geoformas (SGC, 2012).

TABLA 1. Unidades geomorfológicas con sus respectivos ambientes de formación y porcentajes de área. Generada por medio de cálculos de áreas con el ArcGis 10.1.

\begin{tabular}{|c|c|c|c|c|}
\hline Denudativo/Unidades Geomorfológicas & Código & Área $\left(m^{2}\right)$ & $\%$ & $\%$ Ambiente \\
\hline Cono o lóbulo coluvial y de solifluxión & Dco & 46292823,41 & 2,31 & \multirow{7}{*}{6,99} \\
\hline Cono de deslizamiento indiferenciado & Ddi & 1412435,891 & 0,07 & \\
\hline Escarpe de erosión mayor & Deem & 9033222,979 & 0,45 & \\
\hline Lomo denudado bajo largo & Dldebl & 6288037,282 & 0,31 & \\
\hline Ladera erosiva & Dle & 1049228,917 & 0,05 & \\
\hline Lomeríos poco disectados & Dlpd & 17688754,83 & 0,88 & \\
\hline Sierra denudada & Dsd & 58092508,24 & 2,90 & \\
\hline Fluvial/Unidades Geomorfológicas & Código & Área $\left(m^{2}\right)$ & $\%$ & $\%$ Ambiente \\
\hline Abanico fluviotorrencial & Faa & 2861065,53 & 0,14 & \multirow{8}{*}{1,31} \\
\hline Cauce aluvial & Fca & 702720,54 & 0,04 & \\
\hline Cono de deyección & Fcdy & 3213498,111 & 0,16 & \\
\hline Planicie aluvial confinada & Fpac & 472027,3356 & 0,21 & \\
\hline Plano o llanura de inundación & Fpi & 4278505,387 & 0,52 & \\
\hline Terraza de acumulación & Fta & 10493020,88 & 0,12 & \\
\hline Escarpe de terraza de acumulación & Ftae & 2444053,357 & 0,02 & \\
\hline Terraza de acumulación subreciente & Ftas & 1721166,778 & 0,09 & \\
\hline Glacial/Unidades Geomorfológicas & Código & Área $\left(\mathrm{m}^{2}\right)$ & $\%$ & $\%$ Ambiente \\
\hline Circo glaciar y de nivación & $\mathrm{Gc}$ & 827405,4243 & 0,04 & \multirow{17}{*}{35,67} \\
\hline Cuesta estructural glaciada & Gce & 23442495,21 & 1,17 & \\
\hline Conos glaciofluviales & Gcgf & 42205495,7 & 2,11 & \\
\hline Cono o lóbulo de gelifracción & Gclg & 9611207,378 & 0,48 & \\
\hline Espolón estructural glaciado & Gee & 115811169,1 & 5,79 & \\
\hline Espinazo glaciado & Geg & 10465819,99 & 0,52 & \\
\hline Flancos de valle Glaciar & Gflv & 10559987,12 & 0,53 & \\
\hline Laguna Glaciar & Glg & 152895,1244 & 0,01 & \\
\hline Morrena de fondo & Gmf & 3482773,552 & 0,17 & \\
\hline Planos y conos de sobrelavado glaciar & Gpcs & 33618622,59 & 1,68 & \\
\hline Plano Glaciolacustrino & Gpgl & 5770611,149 & 0,29 & \\
\hline Ladera contrapendiente sierra anticlinal glaciada & Gsalc & 71828203,52 & 3,59 & \\
\hline Ladera estructural sierra anticlinal glaciada & Gsale & 66913906,47 & 3,35 & \\
\hline Sierra glaciada & Gsg & 71168256,95 & 3,56 & \\
\hline Sierra homoclinal glaciada & Gshg & 33138181,65 & 1,66 & \\
\hline $\begin{array}{l}\text { Ladera en contrapendiente de sierra homoclinal } \\
\text { glaciada }\end{array}$ & Gshlc & 86048561,69 & 4,30 & \\
\hline Ladera estructural de sierra homoclinal glaciada & Gshle & 128401458,1 & 6,42 & \\
\hline
\end{tabular}


Continuación TABLA 1.

\begin{tabular}{lcccc}
\hline Estructural/Unidades Geomorfológicas & Código & Área $\left(\mathbf{m}^{\mathbf{2}}\right)$ & $\mathbf{\%}$ & $\mathbf{\%}$ Ambiente \\
\hline Cornisa estructural & Scor & 735464,217 & 0,04 & \\
Espolón faceteado & Sefc & 130544296,9 & 6,53 & \\
Espolón faceteado alto de longitud larga & Sefcal & 152716,5578 & 0,01 & \\
Espolón festoneado & Sefesal & 10119902,46 & 0,51 & \\
Espolón & Ses & 89815680,21 & 4,49 & \\
Faceta triangular & Sft & 15859178,12 & 0,79 & \\
Gancho de flexión & Sgf & 9546509,386 & 0,48 & \\
Ladera escalonada & Sles & 24095978,48 & 1,20 & \\
Lomos de falla & Slf & 17035555,98 & 0,85 & \\
Escarpe de línea de falla & Slfe & 43528399,76 & 2,18 & \\
Sierra estructural & Ss & 869384,2607 & 0,04 & \\
Sierra anticlinal & Ssan & 19197076,85 & 0,96 & \\
Sierra homoclinal & Ssh & 34701134,08 & 1,74 & \\
Ladera de contrapendiente de sierra homoclinal & Sshlc & 157433071,6 & 7,87 & \\
Ladera estructural de sierra homoclinal & Sshle & 333139734,5 & 16,66 & \\
Sierra y lomos de presión & Sslp & 5945447,517 & 0,30 & \\
Sierra sinclinal & Sss & 5725396,688 & 0,29 & \\
Ladera de contrapendiente de sierra sinclinal & Ssslc & 147587684,8 & 7,38 & \\
Ladera estructural de sierra sinclinal & Sssle & 74478312,85 & 3,72 & \\
\hline Totales & - & $\mathbf{2 0 0 0 0 0 1 0 4 5}$ & $\mathbf{1 0 0}$ & $\mathbf{1 0 0}$ \\
\hline
\end{tabular}

\section{Aspectos geomorfológicos generales y marco geológico-tectónico de la región}

El departamento de Santander se encuentra ubicado desde el punto de vista geológico en la Provincia Litosférica Continental Mesoproterozoica Grenvilliana de acuerdo con el Servicio Geológico Colombiano según el Mapa Geológico de Colombia; y abarca el cinturón orogénico de la Cordillera Oriental y el valle intercordillerano del Magdalena. Dentro de este contexto regional, la plancha 121 - Cerrito se encuentra en el flanco occidental de la Cordillera Oriental en el Macizo de Santander (MS) definiendo MS como un complejo de rocas ígneas y metamórficas en mayor cantidad a lo largo de su cobertura dentro de los departamentos de Santander y Norte de Santander y en menores cantidades formaciones de roca sedimentaria. Para una mejor comprensión del área estudiada se consideraron tres franjas importantes: Oriental, Central y Occidental. Estas franjas están limitadas al oeste por la Falla de Bucaramanga y al este por el curso del río Servitá.

La franja oriental corresponde a los plegamientos de la Formación Barco, Formación Los Cuervos, Formación
Mirador y Formación Carbonera del Paleógeno y la Formación Colón Mito Juan del Cretáceo, los cuales han sido cortados por la Falla de Servitá. Esta zona abarca parte de la jurisdicción de los municipios de Concepción, Santander y el corregimiento de Presidente, perteneciente al municipio de Chitagá del departamento de Norte de Santander, y se caracteriza por presentar gran diversidad de elevaciones del terreno. Sus drenajes principales son los ríos Servitá y sus afluentes, los cuales forman patrones de drenaje sub-dendrítico. Geológicamente en esta región afloran rocas del Paleógeno representadas por las formaciones Barco, Los Cuervos y Mirador, que se componen mayoritariamente de areniscas, lodolitas y shales. Existe desarrollo de suelos residuales principalmente en las Formaciones Los Cuervos y Mirador. Sus rocas han sido afectadas en grados de meteorización que varían desde moderados a altos. Geomorfológicamente, el ambiente es predominantemente tectónico, el cual está controlado estructuralmente por la falla de Servitá y las principales estructuras de deformación en el paquete sedimentario, que para el área son el Sinclinal de Delgadito y el Anticlinal Vaca Ancha, los que dan lugar a una agrupación de unidades como laderas de contrapendiente fuertemente plegadas. 
La franja central corresponde a la zona más elevada topográficamente, la cual supera los $4.000 \mathrm{msnm}$ y abarca parte de la jurisdicción de los municipios de San Andrés, Baraya, Guaca y Cerrito, en el departamento de Santander. Esta zona es drenada por el río Guaca, la quebrada Lisgaura, el río Sisota y el río Angostura, cuyos afluentes se distribuyen formando patrones de drenaje sub-dendrítico. Geológicamente, esta zona presenta rocas sedimentarias meteorizadas de edad Triásica, Jurásica y Cretácica. En este sector afloran rocas de las Formaciones Girón y Bocas, y parte de la secuencia del Grupo Uribante. Estas rocas desarrollan suelos residuales producto de los procesos de intensa meteorización y erosión. Geomorfológicamente, esta franja presenta predominio glacial, y se pueden apreciar geoformas de sierras glaciadas, valles glaciales, laderas de contrapendiente y laderas estructurales glaciadas. En el ambiente estructural hay buena expresión de geoformas como sierra sinclinal, laderas estructurales y de contrapendiente de sierra sinclinal y anticlinal.

La franja occidental se localiza sobre el costado oeste del río Chicamocha y es la zona más baja y homogénea en términos topográficos. Su máxima altura no supera los $2.500 \mathrm{msnm}$. La región comprende gran parte de los municipios de Piedecuesta, San Andrés y Cepitá en el departamento de Santander. Los principales ríos que bañan esta área son el río Perchiquéz y el río Manco, cuyos afluentes dan lugar a redes de drenaje que varía de tipo sub-dendrítico a dendrítico. La Geología de esta área se compone de rocas de edad Mesoproterozoico, como los neises del Complejo de Bucaramanga y del Ortogneis de Berlín, la Formación Floresta con edad Devónica, y de la Cuarzomonzonita de Santa Bárbara con edad Jurásica. El ambiente geomorfológico predominante es el estructural, aunque hay algunos sectores denudativos y esporádicas zonas con ambiente fluvial. El ambiente fluvial ha sido labrado por procesos de acumulación de los cauces del río Chicamocha y del río Perchiquéz, dando lugar a geoformas como cauce aluvial activo, planicie o llanura de inundación y de terraza aluvial sub-creciente.

Por otra parte, como lo indica Ward et al. (1973), las geoformas de origen denudativo presentes en esta franja son sierras denudadas y escarpes de erosión mayor, las cuales son el resultado de procesos de erosión intensa que afectaron de forma diferencial los macizos rocosos intensamente meteorizados de una superficie originalmente fracturada. No obstante, las unidades geomorfológicas de origen estructural aparecen como vestigios de procesos de fallamientos más antiguos, como es el caso de la Falla de Bucaramanga, que forma laderas estructurales y de contrapendiente $\mathrm{y}$, al mismo tiempo espolones, facetas y con mayor concurrencia laderas acordes con estructuras homoclinales.

\section{Aspectos morfodinámicos de la plancha 121 - Cerrito}

Uno de los procesos geológicos más importantes que ha intervenido en el modelamiento del paisaje en la plancha 121 ha sido el levantamiento de la Cordillera Oriental. En la plancha se presentan cotas de altura de entre los $4.400 \mathrm{msnm}$ en el cinturón plegado y fallado de la Cordillera Oriental en los sectores del páramo de Almorzadero, hasta los $600 \mathrm{msnm}$ en márgenes del río Chicamocha en la esquina suroeste de la plancha (ver MATERIAL SUPLEMENTARIO). El evento orogénico desarrollado durante el Neógeno, resultado del ajuste de la subducción de la placa de Nazca con el margen noroccidental de Suramérica durante este lapso (Chicangana y Vargas, 2013), levanta la cordillera y exhuma al oeste de la plancha un bloque de basamento antiguo constituido por neises del Complejo de Bucaramanga y el Ortogneis de Berlín, la Formación Silgará y algunos cuerpos intrusivos como la Cuarzomonzonita de la Corcova y Santa Bárbara, y el Granito de Pescadero. Como parte de la secuencia sedimentaria aflorante en la parte central y oriental, se encuentran las unidades estratigráficas de la secuencia del Valle del Magdalena Medio y el Catatumbo con rocas que van desde el Triásico hasta el Paleógeno. Los procesos endógenos involucrados en el levantamiento de la Cordillera Oriental generaron expresiones morfológicas caracterizadas por salientes topográficas de origen estructural y denudativo, que a su vez presentan condiciones de relieve con pendientes que pueden alcanzar los $70^{\circ}$ y relieves relativos de entre los $200 \mathrm{~m}$ y $1.500 \mathrm{~m}$. Además de estas condiciones, hay que considerar el fuerte control estructural ejercido por fallas regionales como las de Alto de Guaca, Baraya, Bucaramanga, Morro Las Peñas, Perchiquéz, Pescadito, Servitá, Socotá y Umpalá. Todas ellas generan una alta densidad de fracturamiento y su cinemática ha dado lugar a pliegues de carácter regional tales como el Sinclinal de Angostura, Sinclinal Delgadito, Sinclinal de Almorzadero, Sinclinal El Coronal y el Anticlinal Vaco Ancho. 
Esta evaluación se cuantifica con 58 puntos registrados sobre la plancha 121 - Cerrito, los cuales corresponden a movimientos inventariados y datos obtenidos de la base nacional del Sistema de Información de Movimientos en Masa (SIMMA) (ver FIGURA 2). El tipo de movimiento predominante es el deslizamiento con un $83 \%$, seguido por caídas con $12 \%$ y, en menor proporción, flujos con 5\%. Este comportamiento se ve reflejado con un $22 \%$ de densidad de puntos registrados en la Cuarzomonzonita de Santa Bárbara. De esta manera se argumenta el alto grado de susceptibilidad por movimientos en masa de tipo deslizamiento. Los demás tipos de movimiento se distribuyen sobre rocas sedimentarias que han sufrido fuertes procesos de meteorización física y fracturamiento como las areniscas de la Formación Aguardiente de edad cretácea con un $16 \%$, seguidas por diferentes depósitos, principalmente los de origen coluvial que se han reactivado por agentes detonantes con un $12 \%$ de movimientos registrados, y otras unidades que forman parte de la secuencia Cretácica del Valle Medio del Magdalena con porcentajes que oscilan entre 5 y $10 \%$.

\section{TIPO DE MOVIMIENTO}

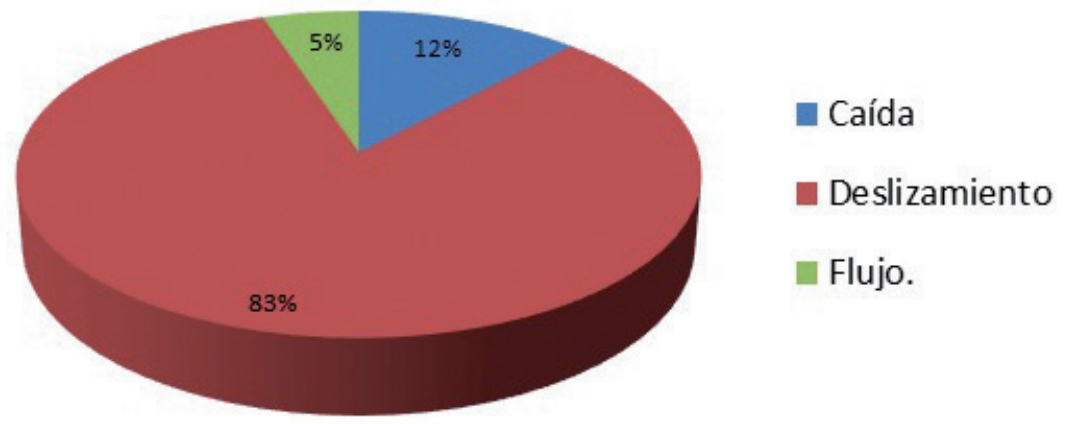

FIGURA 2. Distribución de densidad de movimientos en masa inventariados según su tipo o mecanismo.

\section{Evolución geomorfológica del área del trabajo}

La plancha 121 - Cerrito presenta una compleja actividad tectónica a lo largo del tiempo geológico, que ha estado dominada por eventos de deformación frágil afectando rocas de basamento cristalino del Complejo de Bucaramanga en la parte occidental de la plancha. La zona oriental se encuentra caracterizada por presencia de plegamientos del tipo anticlinal y sinclinal de magnitudes regionales, afectando rocas del Paleógeno predominando en estos, una densidad de fracturamiento alta asociada con la Falla de Servitá y las estructuras regionales del Sinclinal de Almorzadero y el Anticlinal de Vaco Ancho. Debido a esta condición estructural, la generación de suelo es considerable y la consolidación de carácter medio, presenta ausencia de erosión y baja meteorización de minerales arcillosos.

Según Royero y Clavijo (2001), esta dinámica ha permitido la formación de estructuras regionales preCretácicas como la falla de Bucaramanga, que es la responsable del levantamiento de un gran bloque oriental ígneo-metamórfico pre-Devónico conocido como el Macizo de Santander, sobre un bloque occidental conformado por una secuencia sedimentaria Cretácica plegada. Las principales evidencias morfotectónicas de esta zona de falla corresponden a espolones bien marcados sobre la Cuarzomonzonita de Santa Bárbara y la Formación Silgara. Por otra parte, las unidades geomorfológicas presentes en la franja oriental sobre rocas del Paleógeno están definidas como estructuras plegadas donde se exponen los flancos con baja meteorización y los rasgos de estratificación bien marcada. Además de esto, las capas de suelo residual son bajas en estos sectores, lo que genera un mayor desarrollo de deslizamientos traslacionales y flujos locales en los municipios de Cerrito y Concepción. Las épocas de lluvia son otro factor importante dentro del modelado del terreno, ya que genera rasgos erosivos como surcos y algunos casos extremos carcavamiento intenso. Las sierras homoclinales glaciadas resaltando en la parte central acorde a unidades Cretáceas, 
principalmente areniscas bien consolidadas de la Formación Aguardiente y rocas Jurásicas de las Formaciones Girón y Jordán. Estas últimas unidades presentan características como densidad de fracturamiento homogénea con características bajas y medias, siendo afectadas por fallas regionales como la Falla de Baraya, la Falla Perchiquéz y un pequeño tramo de la Falla Angostura. La consolidación de suelos en esta área es alta debido a la baja meteorización de minerales arcillosos y la poca erosión.

En el sector oriental al mismo tiempo encontramos la Falla de Servitá en los municipios de Concepción y Cerrito. Esta estructura regional nos muestra un límite para los plegamientos encontrados hacia el este de la plancha, y para las unidades glaciares representativas del área central de la plancha con presencia de unidades moldeadas en primera instancia por procesos tectónicos (morfoestructurales). Por otra parte, como lo indica Villota (1991), en instancias avanzadas y gracias al levantamiento orogénico se puede observar la generación de masas de hielo en grandes cantidades y generación de valles moldeadas por estas grandes masas. Estas unidades resaltan los movimientos superficiales en capas de suelo con altos contenidos de material orgánico, y presentan deslizamientos traslacionales predominantemente y algunas eventuales caídas de rocas (ver FIGURA 3).
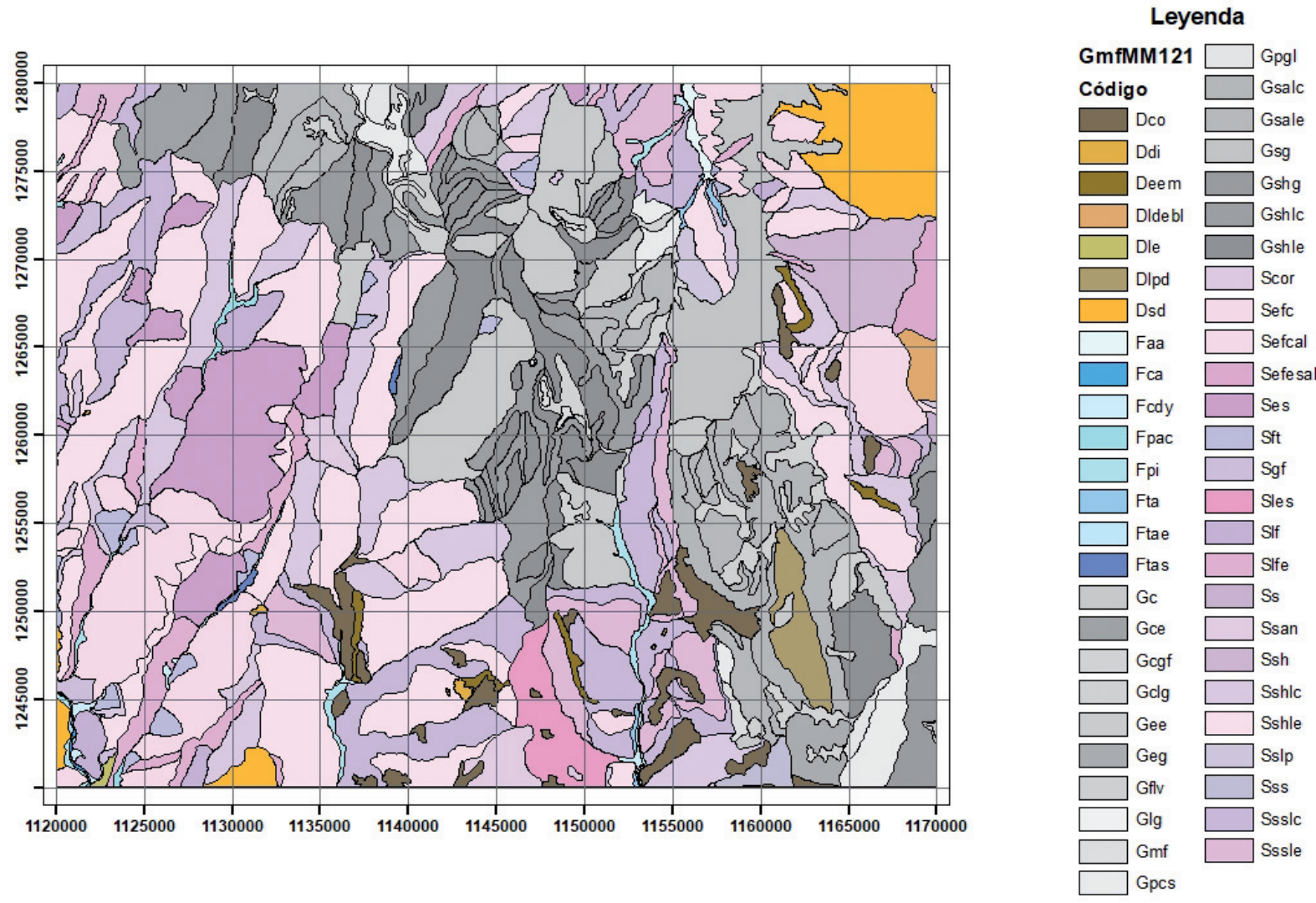

FIGURA 3. Mapa de unidades geomorfológicas de la plancha 121 - Cerrito en el departamento de Santander - Norte de Santander, Colombia, elaborado por medio de ArcGis 10.1 .

\section{DISCUSIÓN}

Con la caracterización e identificación de unidades geomorfológicas a escala 1:100.000, se desarrolla un primer paso para el análisis de la zonificación de las condiciones actuales del terreno relacionado con MM. Encontrar una relación entre las unidades geológicas con su evolución tectónica y con las unidades geomorfológicas fue fundamental para definir cuáles son las zonas susceptibles de MM y que relación tienen con las fallas activas de la región.

La evolución geomorfológica de la plancha 121 - Cerrito de acuerdo con el estudio actual es importante para la diferenciación de subregiones a partir de las características geomorfológicas específicas, la cual siempre está relacionada con sus características geológicas. Para el área de Cerrito, hay que precisar la identificación de 
MM con distribuciones variadas por toda el área de cobertura con características representativas como tipo de movimiento y tipo de material, según la clasificación de Cruden y Varnes (1996), y que fue adoptada por Proyecto Multinacional Andino (PMA, 2007). Con lo anterior se aclara que la caracterización e identificación de unidades geomorfológicas aplicadas a MM es una variable necesaria para continuar con un estudio más avanzado y específico hacia la zonificación de amenaza y susceptibilidad por MM. Por esta razón los datos presentados en este trabajo se consideran de carácter indispensable para la realización de estudios posteriores.

Dentro de los resultados es importante reconocer que debido a su escala intermedia cuando se aplica para escalas más detalladas, se vuelve necesario generar o implementar un cambio de escala a 1:50.000, 1:25.000 o superiores. A esto se lo denomina jerarquización geomorfológica según Verstappen y Van Zuidam (1991), la cual fue adoptada por Carvajal $(2002,2012)$ Carvajal et al. (2003a, 2003b), con el fin de generar una nueva caracterización e identificación de unidades geomorfológicas, dándole mayor ajuste a los contactos entre ambientes geomorfológicos y las unidades de cada tipo. Continuando con el mejoramiento, es importante detallar la geología local (1:25.000), debido a que los mapas se encuentran en condiciones limitadas por los errores de instrumentos de medida durante la época en la que se generaron y debido a nuevos cambios por actividad geomorfológica constante.

\section{CONCLUSIONES}

La identificación de los cuatro ambientes geomorfológicos característicos de la región, y la importancia de cada uno en relación con el tema de movimientos en masa, definen el riesgo y la susceptibilidad de algunas zonas ante procesos constantes y en algunos casos, de grandes magnitudes de remoción en masa. Actualmente el modelado paisajístico del área de estudio se ve influenciado hasta en un $60 \%$ por la variación del clima y los procesos morfodinámicos activos.

La morfodinámica, como parte de la geomorfología, nos ayuda a identificar todo lo relacionado con procesos activos que no solo modifican el paisaje, sino que también ayuda a catalogar sus propiedades mecánicas y las posibles consecuencias de estas. Las características de los suelos de la región juegan un papel sumamente importante dentro de la evolución de toda la geomorfología, brindando una idea de cómo será la generación de nuevas capas de suelo y las condiciones de formación que se sustentan en las propiedades mecánicas del suelo.

De acuerdo con los resultados de esta investigación, encontramos los sectores representativos ante la generación de movimientos en masa, sus geometrías y la Geología del macizo rocoso en el que se están produciendo. Con esto último, se puede hacer una estadística de las unidades litológicas con mayores problemáticas ante la generación de MM.

Desde el punto de vista del desarrollo geotécnico y geomorfológico, este trabajo nos brinda un primer paso para elaborar una zonificación de amenaza y susceptibilidad de la zona de estudio a escala 1:100.000, para poder mejorar la escala geomorfológica y pasar a la creación de estudios 1:50.000 y 1:25.000 tomando como base los mapas generados en este trabajo.

Las unidades geomorfológicas se relacionan con sus respectivas unidades geológicas por medio del bloque diagrama, esto para definir una evolución geomorfológica clara basada en la Geología Regional definida por Royero y Clavijo (2001) para la región de la Provincia de García Rovira, específicamente la plancha de Cerrito escala 1:100.000.

\section{AGRADECIMIENTOS}

A la Universidad Industrial de Santander, y especialmente al Grupo de Zonificación de Amenazas del Área de Geoamenazas del Servicio Geológico Colombiano por la capacitación y el tiempo dedicado para generar los resultados de este trabajo. También agradecemos al profesor Germán Chicangana coordinador de investigación de la Facultad de Ingeniería Civil de la Universidad Santo Tomás, sede Villavicencio, por sus sugerencias para mejorar el contenido y presentación del manuscrito.

\section{REFERENCIAS}

Carvajal, J.H.(2002). Caracterización de la metodología geomorfológica adaptada por INGEOMINAS. Documento interno INGEOMINAS sometido a discusión y modificaciones. Bogotá, Colombia.

Carvajal, J.H. (2012). Propuesta de estandarización de la cartografía geomorfológica en Colombia. Bogotá: Servicio Geológico Colombiano. 
Carvajal, J.H., Carrillo, E., y Bernal, L. (2003a). Documentación detallada del modelo de datos para la faceta de geomorfología. Documento INGEOMINAS preliminar, sometido a discusión y modificaciones. 48p. Medellín, Colombia.

Carvajal, J.H., Carrillo, E., y Bernal, L. (2003b). Visión integral de la geomorfología colombiana. IX Congreso Colombiano de Geología. Medellín, Colombia.

Chicangana, G., and Vargas, C.A. (2013). The subduction geometry change under Colombia and orogenic evolution of the northern Andes in late Neogene times. Acta Geológica Sínica (English Edition), 87(Supp.), 116-118.

Cruden, D.M., and Varnes, D.J. (1996). Landslide types and processes. In: A.K. Turner, R.L. Schuster (eds.). Landslides: investigation and mitigation (pp. 36-75). Transportation Research Board, Special report. vol. 247.

IDEAM. (2014). Cartografía Básica IGAC. Atlas interactivo - Climatológico de Colombia. Grupo de Climatología y Agrometeorología Subdirección de Meteorología - IDEAM. Consultado el 25 de febrero del 2019. http://atlas.ideam.gov.co/ visorAtlasClimatologico.html

PMA. (2007). Una guía para la evaluación de amenazas. Proyecto Multinacional Andino: Geociencias para las Comunidades Andinas. Gobierno de Canadá. Servicio Nacional de Geología y Minería SERNAGEOMIN, 4, $432 \mathrm{p}$.

Royero, J., y Clavijo, J. (2001). Mapa geológico generalizado del departamento de Santander, Escala 1:400.000. Memoria explicativa. INGEOMINAS, Bogotá, Colombia.
SGC. (2012). Propuesta metodológica sistemática para la generación de mapas geomorfológicos analíticos aplicados a la zonificación de amenaza por movimientos en masa escala 1:100.000. Subdirección de Amenazas Geológicas y Entorno Ambiental, Servicio Geológico Colombiano, Bogotá, Colombia. 88p.

Verstappen, H., y Van Zuidam, R. (1991). The ITC system of geomorphology survey: a basis for the evaluation of natural resources and hazards. Villanueva del Huerva, España: ITC Publication, 10.

Villota, H. (1991). Geomorfología aplicada a levantamientos edafológicos y zonificación física de tierras. Instituto Geográfico Agustín Codazzi, Subdirección de Docencia e Investigación. Bogotá, Colombia.

Ward, D., Goldsmith, R., Cruz, J., y Restrepo, H. (1973). Geología de los Cuadrángulos H-12, Bucaramanga y H-13 Pamplona, Departamento de Santander. Boletín Geológico, 21(1-3), 103120 .

Ward, D., Goldsmith, R., Cruz, J., Jaramillo, L., y Vargas, R., (1977). Geología de la plancha 121 Cerrito. Mapa geológico. INGEOMINAS.

\begin{tabular}{c}
\hline \hline Oscar Enrique Forero-Ospino \\
ORCID: 0000-0001-7572-1150 \\
William Fernando Duarte-Delgado \\
ORCID: 0000-0002-4808-8385 \\
\hline \hline
\end{tabular}

Trabajo recibido: abril 01 de 2018

Trabajo aceptado: abril 08 de 2019 